\title{
Correction to: The Nonlinear Heat Equation on W-Random Graphs
}

\author{
Georgi S. Medvedev
}

\begin{abstract}
We correct and improve the main result in Medvedev, "The nonlinear heat equation on W-random graphs", Arch. Rational Mech. Anal., 212(3), pp. 781-803.
\end{abstract}

\section{Correction to: Arch. Rational Mech. Anal. 212 (2014) 781-803 https://doi.org/10.1007/s00205-013-0706-9}

The last step in the proof of Theorem 3.3 estimating $\mathbb{P}\left(\sup _{t \in[0, T]}\left\|z_{n}(t)\right\|_{2, n}^{2}\right.$ $\left.>C n^{-1}\right)$ is incorrect. A similar, albeit quantitatively different estimate can be obtained upon discretizing the time interval $[0, T]$ and estimating the corresponding probability over appropriately chosen small subintervals of $[0, T]$. However, a stronger result can be obtained in a more elementary way. In this note, we correct and improve Theorem 3.3 and provide the necessary changes in the proof.

Theorem 1. Suppose $W \in \mathcal{W}_{0}, g \in L^{\infty}(0,1) \bigcap W^{1,2}(0,1)$ and $D$ is a Lipschitz continuous function on $\mathbb{R}$. In addition, assume that $W(\cdot, y) \in W^{1,2}(0,1)$ for almost every $y \in(0,1)$.

Then for any $T>0$ the solutions of the initial value problem for the discrete and continuum models (2.1), (2.2) and (2.3), (2.4) satisfy

$$
\lim _{n \rightarrow \infty} n^{\frac{1-\alpha}{4}} \sup _{t \in[0, T]}\left\|u_{n}(t)-\mathbf{P}_{\tilde{X}_{n}} u(x, t)\right\|_{2, n}=0 \text { almost surely, }
$$

where $0<\alpha<1$ is arbitrary.

Corollary 2. If $D$ is a bounded function then the finite time interval of convergence in (1) can be extended to that with $T=\beta \log n$ for some $\beta>0$.

The original article can be found online at https://doi.org/10.1007/s00205-013-0706-9. 
Remark 3. Compared to the original theorem, the improvements are twofold: the assumption (3.2) is no longer used, and convergence in probability is replaced by almost sure convergence.

Remark 4. The size of the time interval in (1) on which the convergence takes place can be extended to $O(\log n)$ without the additional assumption that $D$ is bounded. However, this requires a more careful analysis, which will be presented elsewhere.

Remark 5. We strengthened the assumptions on $W(x, y)$ and $g(x)$ by requiring the existence of weak derivatives with respect to $x$. Under these assumptions, the unique solution of the initial value problem $(2.3),(2.4) u(x, t)$, which apriori is in $C^{1}\left(\mathbb{R}, L^{\infty}(0,1)\right)$, is a continuous function on $[0,1] \times \mathbb{R}$ (cf. [3, Theorem 3.2]). In particular, the values $u\left(x_{i}, t\right), i \in \mathbb{N}, t \in[0, T]$, are well-defined. These extra conditions can be dropped if, instead of evaluating the solution of the continuum problem at points $x_{i}, i \in \mathbb{N}$, (see 1 ) one takes the average value of $u(x, t)$ over small intervals in $x$ (see [2]).

Proof of Theorem 1. The proof proceeds as the proof of Theorem 3.3 up to the differential inequality (3.29):

$$
\frac{\mathrm{d}}{\mathrm{d} t}\left\|\zeta_{n}(t)\right\|_{2, n}^{2} \leq a\left\|\zeta_{n}(t)\right\|_{2, n}^{2}+\left\|z_{n}(t)\right\|_{2, n}^{2},
$$

where $a:=4 L+1>0$. From this point we proceed as in the proof of $[1$, Lemma 1.1]. By Gronwall's inequality,

$$
\sup _{t \in[0, T]}\left\|\zeta_{n}(t)\right\|_{2, n}^{2} \leq e^{a T} \int_{0}^{T} e^{-a s}\left\|z_{n}(s)\right\|_{2, n}^{2} \mathrm{~d} s .
$$

It remains to estimate the integral on the right-hand side of (3).

By the definition of $\|\cdot\|_{2, n}$, for $z_{n}=\left(z_{n, 1}, z_{n, 2}, \ldots, z_{n, n}\right)$ we have

$$
\left\|z_{n}\right\|_{2, n}^{4}=n^{-2}\left(\sum_{i=1}^{n} z_{n, i}^{4}+2 \sum_{1 \leq k<l \leq n} z_{n, k}^{2} z_{n, l}^{2}\right) .
$$

Using the independence of $z_{n, k}^{2}$ and $z_{n, l}^{2}, k \neq l$, and moment estimates (3.13) and (3.14) together with (3.11), we have

$$
\mathbb{E} z_{n, i}(t)^{2}=n^{-1} \sigma^{2}(t)+O\left(n^{-2}\right), \mathbb{E} z_{n, i}(t)^{4}=O\left(n^{-2}\right), \quad i \in[n],
$$

where $\sigma^{2}(t)$ is a bounded on $[0, T]$ function defined in Corollary 3.6 with $f(x, y, t):=$ $D(u(y, t)-u(x, t))$. The combination of (4) and (5) yields

$$
\mathbb{E}\left\|z_{n}\right\|_{2, n}^{4}=n^{-2} \sigma^{4}(t)+O\left(n^{-3}\right),
$$

where $\sigma^{4}(t):=\left(\sigma^{2}(t)\right)^{2}$. 
We now return to estimating the right-hand side of (3). Using Cauchy-Schwarz inequality, we have

$$
\begin{aligned}
\mathbb{E}\left(\int_{0}^{T} e^{-a s}\left\|z_{n}(s)\right\|_{2, n}^{2} \mathrm{~d} s\right)^{2} & \leq \int_{0}^{T} e^{-2 a s} \mathrm{~d} s \mathbb{E}\left(\int_{0}^{T}\left\|z_{n}(s)\right\|_{2, n}^{4} \mathrm{~d} s\right) \\
& \leq(2 a)^{-1} \mathbb{E}\left(\int_{0}^{T}\left\|z_{n}(s)\right\|_{2, n}^{4} \mathrm{~d} s\right) .
\end{aligned}
$$

Using the Fubini theorem and (6), we obtain

$$
\begin{aligned}
\mathbb{E}\left(\int_{0}^{T}\left\|z_{n}(s)\right\|_{2, n}^{4} \mathrm{~d} s\right) & =\int_{0}^{T} \mathbb{E}\left(\left\|z_{n}(s)\right\|_{2, n}^{4}\right) \mathrm{d} s \\
& \leq n^{-2} \int_{0}^{T}\left(\sigma^{4}(s) \mathrm{d} s+O\left(n^{-1}\right)\right) \mathrm{d} s
\end{aligned}
$$

The combination of (7) and (8) yields

$$
\mathbb{E}\left(\int_{0}^{T} e^{-a s}\left\|z_{n}(s)\right\|_{2, n}^{2} \mathrm{~d} s\right)^{2} \leq \frac{C_{1}}{n^{2}},
$$

where $C_{1}=C_{1}(T)$ does not depend on $n$. Using Markov inequality and (9), for any $C_{2}>0$ and $0<\alpha<0.25$ we have

$$
\begin{aligned}
& \mathbb{P}\left(n^{0.5-\alpha} \int_{0}^{T} e^{-a s}\left\|z_{n}(s)\right\|_{2, n}^{2} \mathrm{~d} s>C_{2}\right) \\
& \quad \leq n^{1-2 \alpha} C_{2}^{-2} \mathbb{E}\left(\int_{0}^{T} e^{-a s}\left\|z_{n}(s)\right\|_{2, n}^{2} \mathrm{~d} s\right)^{2} \leq \frac{C_{1} C_{2}^{-2}}{n^{1+2 \alpha}} .
\end{aligned}
$$

By the Borel-Cantelli lemma,

$$
n^{0.5-\alpha} \int_{0}^{T} e^{-a s}\left\|z_{n}(s)\right\|_{2, n}^{2} \mathrm{~d} s \leq C_{2} \quad \text { almost surely. }
$$

By plugging this bound into (3), we have

$$
n^{0.25-\alpha} \sup _{t \in[0, T]}\left\|\zeta_{n}(t)\right\|_{2, n} \leq \frac{\sqrt{e^{a T} C_{2}}}{n^{0.5 \alpha}} .
$$

This concludes the proof.

Proof of Corollary 2. If $D$ is bounded on $\mathbb{R}$ then so is $\sigma^{4}(t)$ in (6), and $C_{1}=O(T)$. Then $T$ can be taken $O(\log n)$ such that the right-hand side of (12) goes to 0 as $n \rightarrow \infty$, while the right-hand side of (10) is still summable.

The new proof supersedes the remainder of the proof of Theorem 3.3 starting from Equation (3.30). Furthermore, aside from the pointwise moment estimates (3.13) and (3.14), the proof does not use any other preparatory estimates developed in Lemma 3.5 and Corollary 3.6. In particular, the new proof does not rely on the Central Limit Theorem.

The changes in the statement and the proof of Theorem 3.3 presented in this note apply verbatim to the statement and the proof of Theorem 4.3. 
Acknowledgements. The author thanks Giambattista Giacomin for pointing out the gap in the proof of Theorem 3.3 and for suggesting the way for improvement. This work was supported in part by the NSF Grant DMS 1715161.

\section{References}

1. Kaliuzhnyi-Verbovetskyi, D., Medvedev, G.S.: The mean field equation for the Kuramoto model on graph sequences with non-Lipschitz limit. SIAM J. Math. Anal., 50(3), 2441-2465 (2018)

2. Medvedev, G.S.: The continuum limit of the Kuramoto model on sparse random graphs. ArXiv e-prints (2018)

3. Medvedev, G.S.: The nonlinear heat equation on dense graphs and graph limits. SIAM J. Math. Anal. 46(4), 2743-2766 (2014)

Georgi S. Medvedev

Department of Mathematics,

Drexel University,

3141 Chestnut Street,

Philadelphia,

PA

19104,

USA.

e-mail: medvedev@drexel.edu

(Received October 10, 2018)

Published online November 5, 2018

(C) Springer-Verlag GmbH Germany, part of Springer Nature (2018) 